\title{
On the limit of some Toeplitz-like determinants
}

\author{
Craig A. Tracy \\ Department of Mathematics and Institute of Theoretical Dynamics \\ University of California, Davis, CA 95616, USA \\ e-mail address: tracy@itd.ucdavis.edu
}

Harold Widom

Department of Mathematics

University of California, Santa Cruz, CA 95064, USA

e-mail address: widom@math.ucsc.edu

Our Toeplitz-like matrices are of the form

$$
M=\left(c_{p_{i}-q_{j}}\right), \quad i, j=0,1, \cdots
$$

where $\left\{p_{i}\right\}$ and $\left\{q_{i}\right\}$ are sequences of integers satisfying $p_{i}=q_{i}=i$ for $i$ sufficiently large, say for $i \geq m$. These are a particular class of finite-rank perturbations of Toeplitz matrices. If the $p_{i}$ and the $q_{i}$ are all different then after rearranging the first $m$ rows and columns these become minors of the Toeplitz matrix $\left(c_{i-j}\right)$ obtained by removing finitely many rows and columns.

The problem is to determine the asymptotics of the determinants of large sections of this matrix and we do this under certain conditions. We assume that $\left\{c_{i}\right\}$ is the sequence of Fourier coefficients of a bounded function $\varphi$, so that $\left(c_{i-j}\right)=T(\varphi)$ in the usual notation. We assume also that $T(\varphi)$ is invertible on the space $\ell^{2}\left(\mathbf{Z}^{+}\right)$, and that is almost all. (We shall explain this below.)

For convenience in notation we consider the $(m+n) \times(m+n)$ sections of $M$,

$$
M_{m+n}=\left(c_{p_{i}-q_{j}}\right), \quad i, j=0,1, \cdots, m+n-1,
$$

and denote by $T_{n}(\varphi)$ the $n \times n$ Toeplitz matrix $\left(c_{i-j}\right)_{i, j=0,1, \cdots, n-1}$.

If $T(\varphi)$ is invertible then $\varphi$ has a factorization $\varphi=\varphi^{-} \varphi^{+}$where the functions $\left(\varphi^{+}\right)^{ \pm 1}$ and $\left(\varphi^{-}\right)^{ \pm 1}$ belong to $H^{2}$ and $\overline{H^{2}}$ respectively.1 This is the "Wiener-Hopf factorization" of $\varphi$. In terms of these factors (and with subscripts denoting Fourier coefficients) our limit formula is

$$
\lim _{n \rightarrow \infty} \frac{\operatorname{det} M_{m+n}}{\operatorname{det} T_{n}(\varphi)}=\operatorname{det}\left(\sum_{k=1}^{\infty}\left(\varphi^{-}\right)_{p_{i}+k-m}\left(\varphi^{+}\right)_{-q_{j}-k+m}\right)_{i, j=0, \cdots, m-1} .
$$

(The sum in the determinant on the right side of (1) has only finitely many nonzero terms since the Fourier coefficient $\left(\varphi^{-}\right)_{k}$ vanishes for $k>0$ and $\left(\varphi^{+}\right)_{k}$ vanishes for $k<0$.)

\footnotetext{
${ }^{1}$ Recall that $H^{2}$ consists of the $L^{2}$ functions whose Fourier coefficients with negative index all vanish. The sequences of Fourier coefficients with nonnegative indices of $\varphi^{+}$and $\overline{\varphi^{-}}$are, up to constant factors, $T(\varphi)^{-1} \delta$ resp. $T(\bar{\varphi})^{-1} \delta$ where $\delta$ is the sequence $\{1,0,0, \cdots\}$.
} 
Under some additional hypotheses the strong Szegö limit theorem gives the asymptotics of the Toeplitz determinant:

$$
\operatorname{det} T_{n}(\varphi) \sim G(\varphi)^{n} E(\varphi)
$$

where

$$
G(\varphi)=\exp \left\{\frac{1}{2 \pi} \int \log \varphi(\theta) d \theta\right\}, \quad E(\varphi)=\exp \left\{\sum_{k=1}^{\infty} k(\log \varphi)_{k}(\log \varphi)_{-k}\right\}
$$

Our result holds without these extra hypotheses. What we do need, which is a little stronger than the invertibility of $T(\varphi)$, is the uniform invertibility of the $T_{n}(\varphi)$. That is, we require that the $T_{n}(\varphi)$ be invertible for sufficiently large $n$ and that the norms of the inverse matrices $T_{n}(\varphi)^{-1}$ be bounded as $n \rightarrow \infty$. This holds in all "normal" cases where $T(\varphi)$ is invertible. The known counterexamples are not simple. (For a discussion of these points see [2], especially Chap. 2.) If $\varphi$ has a continuous logarithm, for example, then the $T_{n}(\varphi)$ are uniformly invertible.

Theorem. If the $T_{n}(\varphi)$ are uniformly invertible then (11) holds.

Proof. We use the fact from linear algebra that if we have a matrix

$$
M=\left(\begin{array}{ll}
A & B \\
C & D
\end{array}\right)
$$

with $A$ and $D$ square and if $D$ is invertible then

$$
\operatorname{det} M=\operatorname{det} D \operatorname{det}\left(A-B D^{-1} C\right) .
$$

In our case $M=M_{m+n}, A=\left(c_{p_{i}-q_{j}}\right)_{i, j=0, \cdots, m-1}$ and $D$ is the Toeplitz matrix $T_{n}(\varphi)$. If the indices for $B$ and $C$ start at 0 then their entries are given by

$$
B_{i, k}=c_{p_{i}-m-k}, \quad C_{k, j}=c_{m+k-q_{j}}, \quad(i, j=0, \cdots, m-1, \quad k=0, \cdots, n-1),
$$

and we are interested in the limit as $n \rightarrow \infty$ of the $i, j$ entry of the $m \times m$ matrix $B T_{n}(\varphi)^{-1} C$.

Now we use the fact that if the $T_{n}(\varphi)$ are uniformly invertible then $T_{n}(\varphi)^{-1}$ converges strongly to the infinite Toeplitz matrix $T(\varphi)^{-1}$. (See [2], Prop. 2.2.) It follows that each entry of $B T_{n}(\varphi)^{-1} C$ converges to the corresponding entry of $B T(\varphi)^{-1} C$ where now $B$ and $C$ are the $m \times \infty$ and $\infty \times m$ matrices, respectively, with entries given by (2) but now with $k \in \mathbf{Z}^{+}$. What remains is to show that the $i, j$ entry of $A-B T(\varphi)^{-1} C$ is given by the summand on the right side of (1).

It is convenient to extend the range of the row or column indices of our Toeplitz matrices so that one or the other can run over $\mathbf{Z}$ rather than $\mathbf{Z}^{+}$. So we introduce the notations

\footnotetext{
${ }^{2}$ For such a $\varphi$ the Wiener-Hopf factors $\varphi^{-}$and $\varphi^{+}$are, up to constant factors, the exponentials of the portions of the Fourier series of $\log \varphi$ corresponding to negative resp. positive indices.
} 
$T^{(r)}(\varphi)$ and $T^{(c)}(\varphi)$ for the matrices in which the row resp. the column index runs over $\mathbf{Z}$. With this notation, we see that

$$
B_{i, k}=T^{(r)}(\varphi)_{p_{i}-m, k}, \quad C_{k, j}=T^{(c)}(\varphi)_{k, q_{j}-m}
$$

Thus

$$
\left(B T(\varphi)^{-1} C\right)_{i, j}=\left(T^{(r)}(\varphi) T(\varphi)^{-1} T^{(c)}\right)_{p_{i}-m, q_{j}-m}
$$

Now it is well-known and an easy check (see [2], Prop. 1.13) that

$$
T(\varphi)=T\left(\varphi^{-}\right) T\left(\varphi^{+}\right), \quad T(\varphi)^{-1}=T\left(\varphi^{+}\right)^{-1} T\left(\varphi^{-}\right)^{-1}
$$

and just as easy that

$$
T^{(r)}(\varphi)=T^{(r)}\left(\varphi^{-}\right) T\left(\varphi^{+}\right), \quad T^{(c)}(\varphi)=T\left(\varphi^{-}\right) T^{(c)}\left(\varphi^{+}\right)
$$

It follows that

$$
T^{(r)}(\varphi) T(\varphi)^{-1} T^{(c)}(\varphi)=T^{(r)}\left(\varphi^{-}\right) T^{(c)}\left(\varphi^{+}\right)
$$

Hence

$$
\begin{gathered}
\left(B T(\varphi)^{-1} C\right)_{i, j}=\sum_{k=0}^{\infty}\left(\varphi^{-}\right)_{p_{i}-m-k}\left(\varphi^{+}\right)_{m+k-q_{j}} \\
=\sum_{k=-\infty}^{\infty}\left(\varphi^{-}\right)_{p_{i}-m-k}\left(\varphi^{+}\right)_{k-q_{j}+m}-\sum_{k=-\infty}^{-1}\left(\varphi^{-}\right)_{p_{i}-m-k}\left(\varphi^{+}\right)_{k-q_{j}+m} \\
=\varphi_{p_{i}-q_{j}}-\sum_{k=1}^{\infty}\left(\varphi^{-}\right)_{p_{i}-m+k}\left(\varphi^{+}\right)_{-k-q_{j}+m} .
\end{gathered}
$$

The first term on the right is $A_{i, j}$, and so the theorem is established.

\section{Acknowledgments}

Recently D. Bump and P. Diaconis [1] obtained the asymptotics for the same determinants with an answer having a very different form (although it must be the same as ours) and using very different techniques - the representation theory of the symmetric group. We thank them for alerting us to their results and sending us copies of their manuscript. We also acknowledge support from the National Sciance Foundation through grants DMS-9802122 (first author) and DMS-9732687 (second author).

\section{References}

[1] D. Bump and P. Diaconis, Toeplitz minors, to appear in J. Comb. Th. A.

[2] A. Böttcher, and B. Silbermann, Introduction to large truncated Toeplitz matrices, Springer-Verlag, heidelberg, 1999. 